\title{
Frequency-division multiplexing for multicomponent shearography
}

\author{
Ian A. Bledowski, Thomas O. H. Charrett, Daniel Francis, \\ Stephen W. James, and Ralph P. Tatam* \\ Department of Engineering Photonics, School of Engineering, Cranfield University, Cranfield, Bedfordshire, MK43 OAL, UK \\ *Corresponding author: r.p.tatam@cranfield.ac.uk \\ Received 19 October 2012; accepted 27 November 2012; \\ posted 11 December 2012 (Doc. ID 178396); published 11 January 2013
}

\begin{abstract}
A new method of multiplexing the speckle patterns needed in multicomponent digital shearography systems is presented. Frequency-division multiplexing (FDM) of the measurement channels is achieved by recording speckle patterns from objects illuminated by intensity-modulated sources. Each source is modulated at a discrete frequency, which is less than half of the camera frame rate, and a bank of images of the modulated speckle patterns is captured. This allows for pixel-by-pixel Fourier-based extraction of the individual speckle patterns from peaks in the power spectra. The approach is demonstrated with a two-component in-plane shearography instrument. (c) 2013 Optical Society of America
\end{abstract}

OCIS codes: $\quad 110.4155,120.3940,120.6165,120.4290$.

\section{Introduction}

Shearography [1] is a noncontact full-field optical interferometric technique that allows the measurement of the gradients of displacement on surfaces subjected to loading. This is a property that lends itself well to the measurement of surface strain. It is an interferometric contactless technology that can be used to reveal gradients of deformation across an object's surface in applications ranging from large field-of-view examinations of vibrational modes across aircraft panels [2] to quantitative measurements of strain on microscopic microelectromechanical systems (MEMS) devices [3]. A speckle interferogram is obtained by illumination of an object's surface with a laser. As long as the surface is "rough" on the scale of the wavelength of light, a speckle pattern is produced. The speckle pattern is imaged using an image-shearing device, which is discussed further below, resulting in an interferometric speckle pattern that is sensitive to the surface displacement gradient. Processing the phase changes

$1559-128 \mathrm{X} / 13 / 030350-09 \$ 15.00 / 0$

(C) 2013 Optical Society of America induced in interferograms taken before and after loading the surface produces a result that can be related to surface strain. A system consisting of a single illumination and viewing direction results in a speckle interferogram that, in general, contains contributions from the in-plane and out-of-plane surface displacement gradient components. The relative proportions of these are dictated by the orientation of the sensitivity vector, which is defined as the bisector of the observation and illumination axes. A number of techniques have been developed to enable separation of the in-plane and out-of-plane components, with the general requirement that a minimum of either three illumination directions viewed by a single detector array, or a single illumination direction viewed by three detector arrays, is required to obtain the full, three-dimensional surface strain field $[4,5]$. More recently it has been shown that a fourth measurement channel can result in lower errors in the recovered strain components [ $[\underline{6}]$. A number of techniques to multiplex the three, or more, measurement channels have been reported.

Time-division-multiplexed (TDM) systems that capture the speckle patterns from each channel sequentially, at different points in time relative to 
each other, using a single imaging sensor, have been investigated [5]. Alternatively, by spatial multiplexing (SM), the images can be captured in parallel using multiple observation directions and a single illumination source. This can be done in principle using multiple image-shearing interferometers [7], which makes use of the full spatial resolution of each of the imaging sensors. Alternatively, coherent imaging fiber bundles [8] can port multiple views to a single interferometer, although this technique suffers from a loss of image resolution as the views are imaged to subregions of the sensor. SM requires good pixel registration between images in order to reduce errors [9]. Parallel image capture can also be achieved using multiple illumination directions and a single interferometer exploiting polarizationdivision multiplexing (PDM) [10] or wavelengthdivision multiplexing (WDM) [11]. In these, the channels are separated by use of polarization- or wavelength-selective optics and directed onto image sensors, again utilizing the full spatial resolution of the sensor.

In this paper, a novel configuration for multiplexing the measurement channels exploiting frequencydivision multiplexing (FDM) is described. This allows for the simultaneous capture of speckle patterns from each measurement channel on a single imaging sensor without loss of spatial resolution. The theory of quantitative shearography is summarized, and the application of FDM to shearography is described. Finally, the results of experimentally applying FDM to a two-component shearography system are presented and discussed.

\section{Theory of Quantitative Shearography}

A single-channel shearography system, shown in Fig. 1(a), consists of a single laser source that is used to illuminate the surface of an object being tested, an

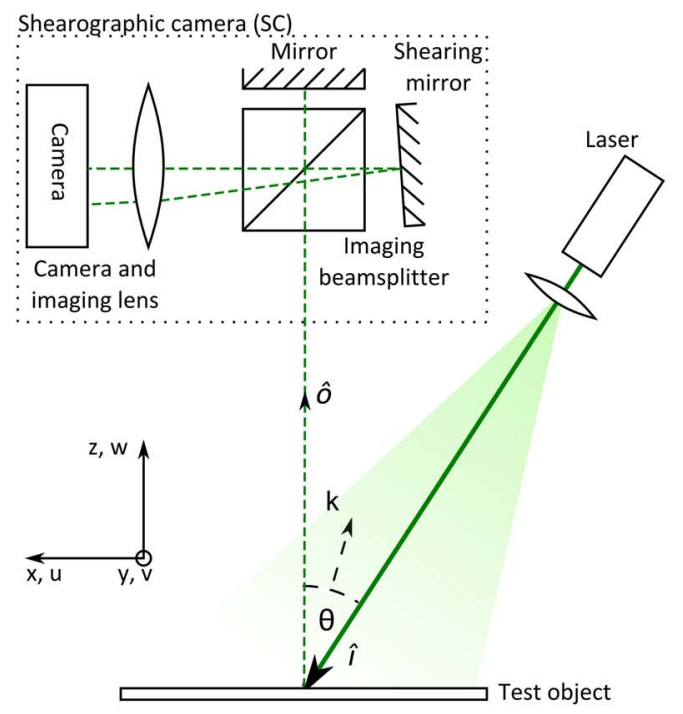

(a)

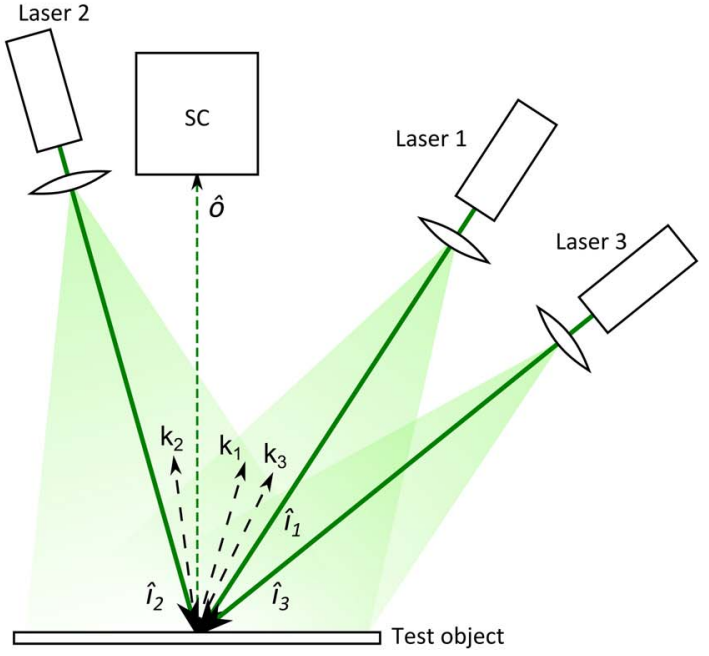

(b)

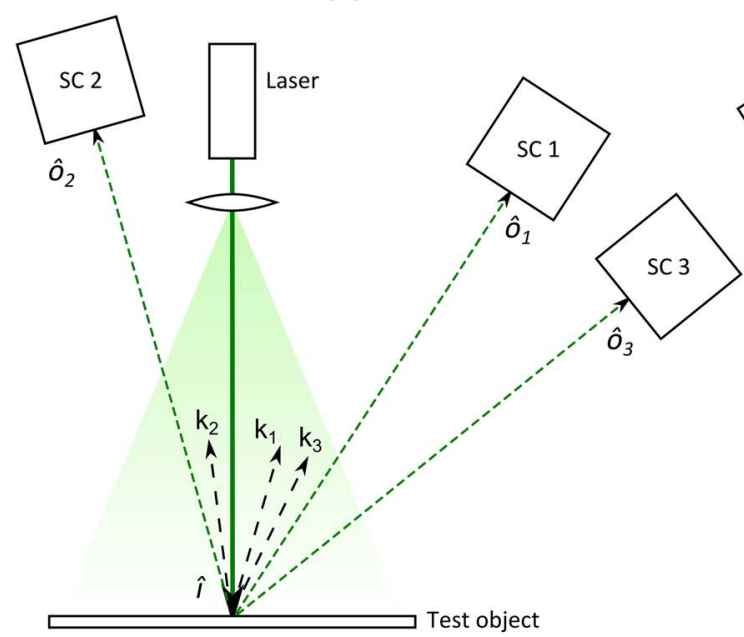

(c)

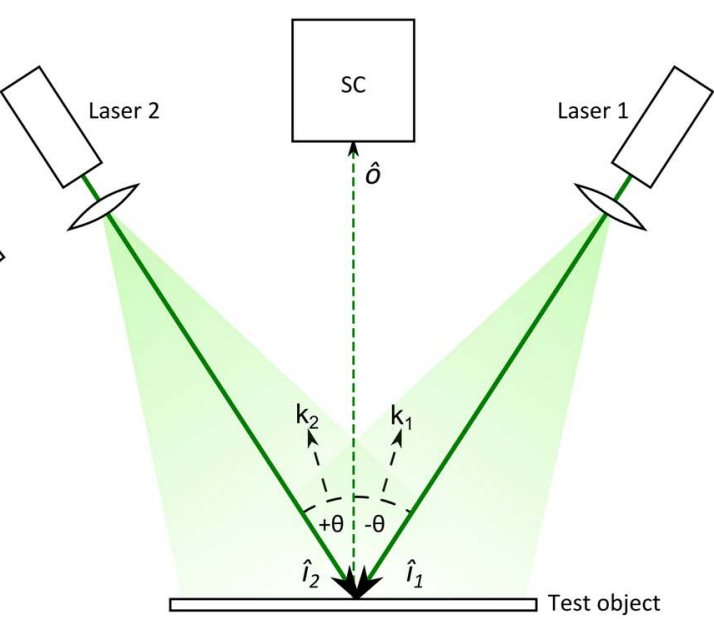

(d)

Fig. 1. (Color online) (a) Representation of a single-channel shearography system and the imaging optics of the shearographic camera (SC, dotted rectangle) [12]. (b) Three-channel system using multiple illumination directions, $\hat{i}$, around a single SC to vary the sensitivity [13]. (c) Three-channel system using multiple observation directions, $\hat{o}$, formed of multiple cameras around a single laser [7]. (d) Two-channel in-plane system with two lasers arranged at equal angles around the observation axis [4]. 
image-shearing device, and an image sensor, collectively referred to as a shearographic camera (SC). Speckle patterns resulting from illumination of the object's surface before and after loading are imaged onto the sensor and are processed to reveal the phase change due to the deformation gradients, $\Delta \phi_{s}$, imposed on the scattered wavefront. The phase change is described in Eq. (1) and shows that the phase is related to the applied image shear, $\delta s$, which can be in either the horizontal $(\partial s=\partial x)$ or the vertical $(\partial s=\partial y)$ direction. The orthogonal surface displacement gradients in the shear direction are denoted as $\partial u / \partial s, \partial v / \partial s$, and $\partial w / \partial s$, and the sensitivity unit-vector components are denoted as $k_{x}, k_{y}$, and $k_{z}$, and the wavelength as $\lambda$ :

$$
\Delta \phi_{s}=\frac{2 \pi \delta s}{\lambda}\left[k_{x} \frac{\partial u}{\partial s}+k_{y} \frac{\partial v}{\partial s}+k_{z} \frac{\partial w}{\partial s}\right]
$$

Therefore, a phase map retrieved from a single channel generally contains contributions from the two in-plane and one out-of-plane displacement gradient terms. From measurements undertaken using a single-channel system, these orthogonal terms are inseparable and so the phase map is generally useful for obtaining only a qualitative indication of the presence and locations of defects within an object, as indicated by a local change in the phase map, and this is the application for which shearography is most used [1].

Quantitative measurements of surface strain require the use of at least three measurement channels, each sensitive to a different combination of the components of the surface displacement gradient [14]. In quantitative shearography, the detectable strain components are obtained through processing phase maps from each measurement channel. Information obtained from multiple phase measurements allows the surface displacement gradient terms to be separated and therefore allows for quantitative measurement of the surface strain components.

Using a system of multiple illumination or observation directions, similar to the two configurations shown in Figs. 1(b) and 1(c), it is possible to obtain quantitative measurement of the three-dimensional surface strain. The channels can be placed arbitrarily to obtain the result using a matrix transform [13], but errors may be improved through a more considered placement of the channels $[1,15]$.

An alternative configuration that can be used to obtain out-of-plane and one in-plane component is shown in Fig. 1(d). Addition and subtraction of phase maps, obtained from two channels positioned under conditions of symmetrical channel placement in a plane defined by the observation and illumination vectors [4], yields the out-of-plane $(\partial w / \partial s)$ and inplane $(\partial \bar{u} / \partial s)$ displacement gradient components. For this configuration $\partial w / \partial s$ and $\partial u / \partial s$ can be found using

$$
\begin{aligned}
& \frac{\partial u}{\partial s}=\frac{\lambda}{4 \pi \delta s k_{x}}\left(\Delta \phi_{1}-\Delta \phi_{2}\right), \\
& \frac{\partial w}{\partial s}=\frac{\lambda}{4 \pi \delta s k_{z}}\left(\Delta \phi_{1}+\Delta \phi_{2}\right),
\end{aligned}
$$

Therefore, in order to obtain quantitative measurements of surface strain, more than one phase must be measured using one of the various multiplexing schemes discussed above.

\section{Frequency-Division Multiplexing}

FDM allows the simultaneous capture of data from each of the channels on a single imaging sensor at full spatial resolution with a common image shear. The technique uses multiple intensity-modulated laser beams and a single shearing interferometer to provide multiple measurement channels. The resulting speckle patterns are therefore received simultaneously at the imaging sensor, and their evolution over time is captured in a sequence of images over a number of modulation cycles. The time series of a given pixel, $I(t)$, will vary in intensity over time as a function of all of the modulation frequencies present:

$$
I(t)=I_{1}(t)+I_{2}(t)+\ldots+I_{n}(t)+C,
$$

where $I_{n}(t)$ is the modulation signal of channel $n$, and $C$ is the background intensity.

To demultiplex the channels, the power spectrum is calculated via the discrete Fourier transform (DFT) on a pixel-by-pixel basis. The peaks corresponding to the different channel modulation frequencies are separated using window functions, and the intensity is evaluated. The root-meansquared (RMS) intensity, $I_{\mathrm{RMS}}$, of a channel's signal can be calculated in the frequency domain using Parseval's theorem:

$$
I_{\mathrm{RMS}}=\sigma_{I_{n}}=\sqrt{\frac{\sum\left|I_{n}(t)\right|^{2}}{N}}=\sqrt{\frac{\sum\left|\mathcal{F}\left\{I_{n}(t)\right\}\right|^{2}}{N^{2}}},
$$

where $\mathcal{F}\left\{I_{n}(t)\right\}$ is the windowed DFT spectrum for the signal, $I_{n}(t)$, and $N$ is the number of samples. As the DC component of the signal is removed when windowing in the frequency domain, the RMS is equivalent to the standard deviation of the signal, $\sigma_{I_{n}}$. Hence, the peak-to-peak intensity, $I_{\mathrm{pp}}$, of the modulating signal can be found by dividing the extracted RMS intensity by the RMS intensity of the normalized waveform, $\sigma_{\mathrm{cal}}$ :

$$
I_{\mathrm{pp}}=\frac{\sigma_{I_{n}}}{\sigma_{\mathrm{cal}}},
$$

This is repeated for each channel present and the values placed into separate image arrays. These images can then be considered equivalent to speckle patterns captured with only a single illumination 


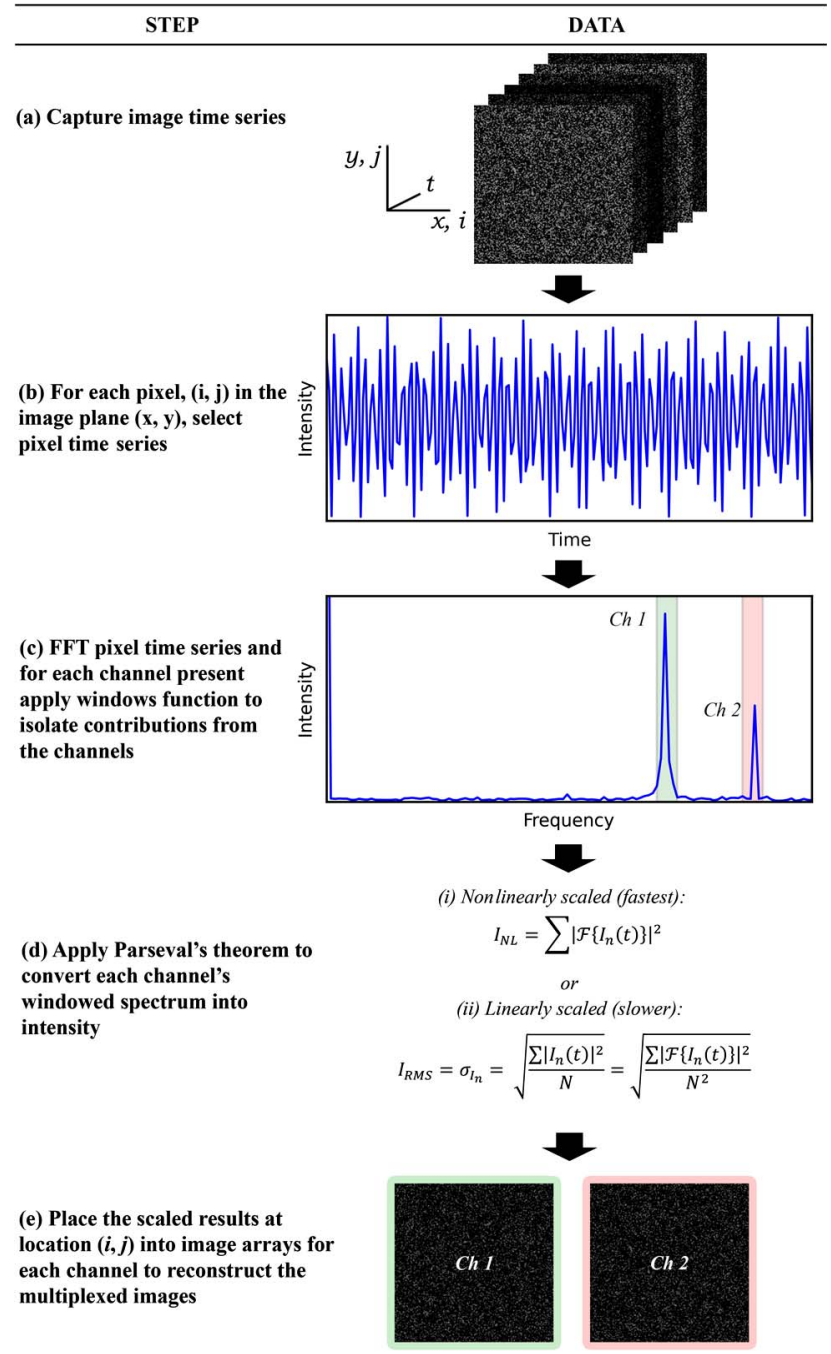

Fig. 2. (Color online) Steps to demultiplex images from an image time series. The example shown is for two-channel demultiplexing.

channel present at any one time. The technique is summarized in Fig. 2, which illustrates the steps of the demultiplexing process.

It should be noted that it is not always necessary to calculate the peak-to-peak amplitudes. Instead, the sum of the windowed power spectrum can be used to speed demultiplexing by omitting steps from the RMS calculation, shown at step (d) part (ii) in Fig. 2 . However, the intensity, $I_{\mathrm{NL}}$, obtained by this method is scaled nonlinearly, which could be a problem when used with some phase-stepping algorithms [16]. Another point to note is that the modulation frequencies should be chosen so that they meet the Nyquist condition and have resolvable peaks in the power spectrum.

\section{Experimental Development}

Development of the processing algorithm was conducted in the open-source Python programming language [17] using the Python Toolkit environment [18], with the NumPy and SciPy [19] modules. A bespoke shared library was then written in $C$ using the FFTW3 library [20] in order to increase memory efficiency and the speed of the DFT-based analysis. This was capable of demultiplexing the channels on the order of seconds using a single core of a desktop PC running at $2.86 \mathrm{GHz}$. Typical data sets captured consist of large, three-dimensional arrays of signals with sizes that can approach 1 Gbytes during processing.

The first step in demonstrating the multiplexing technique was to show that images could be reconstructed and separated from an image bank. An experiment was configured using a high-speed camera (Baumer HXC-13) with a $144 \times 150$ pixel subregion selected on the sensor, allowing a frame rate of $11,000 \mathrm{fps}$. Images were recorded using a field of view of approximately $41 \mathrm{~mm}^{2}$. The expanded beams from two laser sources (a $532 \mathrm{~nm}$ Coherent DPSS 532-300 at $300 \mathrm{~mW}$ and a Photop DPGL-3020 $532 \mathrm{~nm}$ DPSS module at $20 \mathrm{~mW}$ ) were incident on a screen. The beam in each channel was transmitted through a spatial modulator to generate distinct static intensity patterns, these being a calibration target slide and a coiled spring. These patterns were imposed to act as a visual aid to confirm that the algorithm performed as expected.

The outputs from the lasers were intensity modulated in time by placing a beam chopper in each beam path to produce square-wave intensity modulations at rates of 1000 and $1500 \mathrm{~Hz}$ in the respective channels. An image time series of 1024 modulated images was captured, as processing time was not an issue at this stage. After capturing the time series, two single frames were captured with only a single illumination channel present so the demultiplexed result could be assessed against the conventional approach. The time-series images were processed using the technique described above to demultiplex the images from the two channels.

The results from this can be seen in Fig. 3, which shows the single-frame captured images [(a) and (b)] above their demultiplexed counterparts [(c) and (d)]. It can be seen that the images are reconstructed with good fidelity. Also shown are the demultiplexed images when the less computationally intensive method of calculating intensity from the power spectrum is used [(e) and (f)], omitting the scaling process to approach an RMS intensity. These images show clearly that the nonlinear method introduces a more rapid dropoff from high to low intensity in the demultiplexed images, as compared to the single-frame captures. If processing time is an issue (for examplem if attempting to process in real time), then these images may be used with a temporal phasestepping algorithm that is insensitive to nonlinearities [16]. As the time gain was only a fraction of a second, the RMS scaled version of the calculation was adopted for use in postprocessing.

Similar to the above, demultiplexed images can be reconstructed using illumination channels originating from a common laser source, and at a range of camera frame rates and modulation frequencies (provided the Nyquist condition is met). 

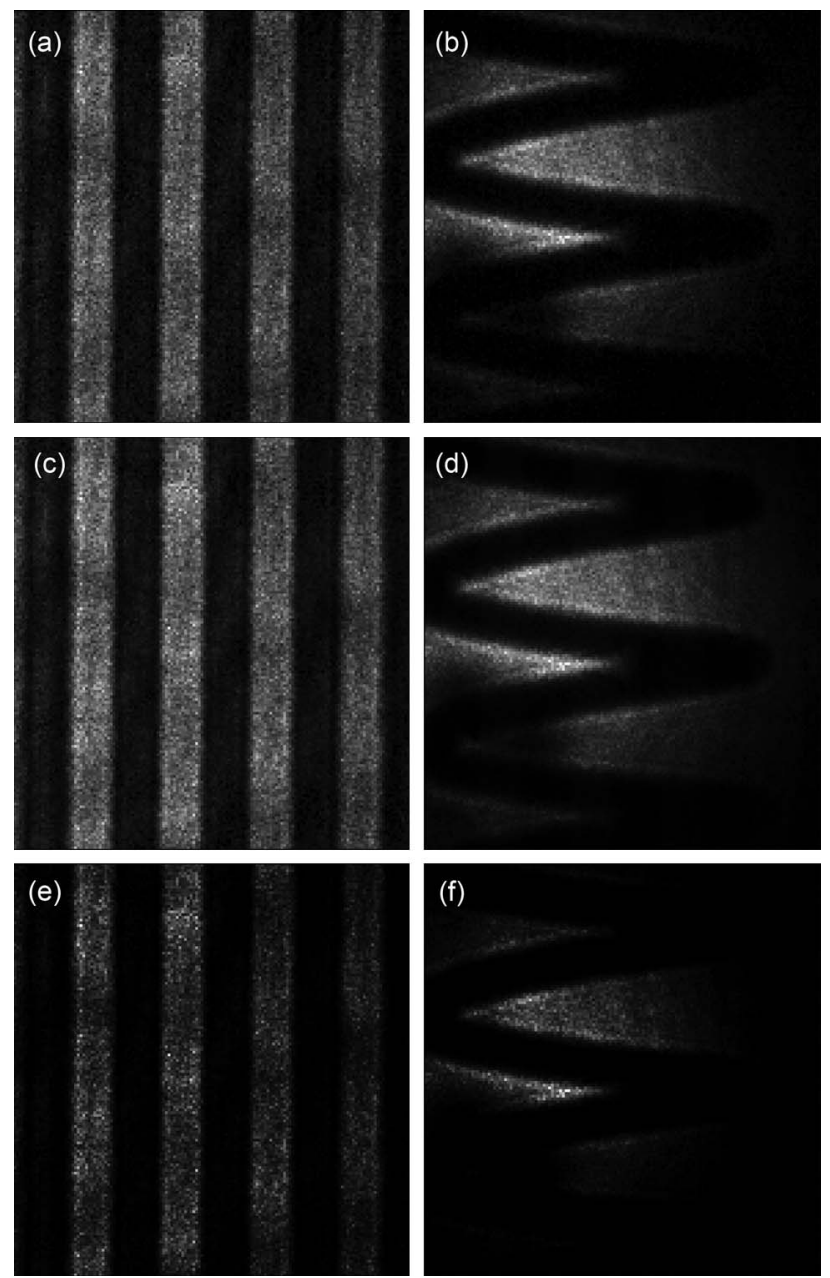

Fig. 3. (a) and (b) Directly captured images of patterns produced from sources in separate channels across a field of view of approximately $4 \mathrm{~cm}^{2}$. (c) and (d) Demultiplexed images of the same patterns that had been multiplexed together by chopping the beams at $1 \mathrm{kHz}$ and $1.5 \mathrm{kHz}$, respectively, and captured in an image time series of 1024 frames at 11,000 fps. Images in (e) and (f) show the same patterns demultiplexed using the nonlinear method where intensity is the sum of the squares of the windowed power spectrum. Note the nonlinear intensity response produced by that method in the demultiplexed images increases more sharply toward the bright regions. Each image is scaled to the highest intensity in the frame.

As the demultiplexing technique effectively filters the power spectrum, the camera noise spectrum will also be filtered, so that the noise remaining in the demultiplexed images is reduced compared with that in a single frame measurement. The camera used in this work (Baumer HXC-13) is a CMOS image sensor; therefore each pixel will have its own noise characteristics described by a mean, $\mu(i, j)$, and standard deviation, $\sigma(i, j)$, where $i$ and $j$ are the pixel array indices. To examine the camera's noise characteristics under the conditions of the experimental demonstration of FDM shearography in this paper, a lens cap was placed over the camera sensor and an image time series of 256 frames was captured at a frame rate of $430 \mathrm{fps}$. From this, two images were demultiplexed using windows centred at 140 and $175 \mathrm{~Hz}$, each of width $10 \mathrm{~Hz}$. The image time series was also averaged along the time axis to produce an image of the average intensity at each pixel over the capture period. This time-averaged image was analyzed so as to investigate whether the demultiplexing method produced improved noise characteristics over simply taking the mean of a time series. The level of the camera noise present in the demultiplexed and the time-averaged images was compared to that of single-frame measurements, equivalent to TDM, one captured at the same time as each time series. The whole procedure was repeated 100 times, giving a set of demultiplexed images from which the means, $\mu(i, j)$, and standard deviations, $\sigma(i, j)$, could be determined at each pixel site over time. The values for the pixels were then averaged across the frame, and the results are presented in Table 1.

Overall, the demultiplexing method was shown to reduce the mean noise floor of the camera compared to both the single-frame and the time-averaged images by a factor of 14 . The consistency of the measurements, shown by the average standard deviation of all the pixels, obtained by demultiplexing, was also greatly improved compared to the single-frame TDM approach, showing an improvement of a factor of 20 .

In comparison to performing a time averaging of the same number of frames, the demultiplexed data standard deviation was less. However, while the FDM technique showed an improvement in the level of the noise floor, the signal-to-noise ratio was half that obtained by time averaging, due to the $50 \%$ duty cycle of the modulation signal used to multiplex the channels. While this may seem to place FDM at a disadvantage relative to the simpler time-averaging approach, it is worth noting that time averaging is only possible on a single channel, whereas the FDM approach allows the capture of multiple images simultaneously in a single time series of the same length. For the same total capture period, $T$, FDM can capture $n$ channels with noise averaging over the whole image time-series capture period (equal to $T$ ), while time averaging would only be able to capture in the space of $T / n$ per channel, greatly reducing the benefits of averaging. This could be of concern when measuring a dynamically evolving system.

A further source of error is the cross talk between measurement channels. This was measured for the situation in which the multiplexed speckle patterns were produced by illuminating a surface with two expanded beams split from a common laser source (Coherent DPSS 532-300). The intensities of the beams were modulated by placing mechanical choppers in the beam paths, resulting in modulation frequencies of 140 and $175 \mathrm{~Hz}$. As before, the camera (Baumer HXC-13) was set to capture the image time series consisting of 256 frames at a frame rate of $430 \mathrm{fps}$. The surface was illuminated by a single modulating channel, each in turn, and a speckle-pattern time series was captured for each. From each time series two speckle patterns were demultiplexed. 
Table 1. Noise Characteristics of an Average Pixel Calculated over 100 Measurements for Single-Frame, Time Averaged, and Demultiplexed Techniques ${ }^{a}$

\begin{tabular}{lcccc}
\hline & Single-Frame & Time Averaged (256 Frames) & Demultiplexed $(f=140 \mathrm{~Hz})$ & Demultiplexed $(f=175 \mathrm{~Hz})$ \\
\hline TPB $\langle\mu(i, j)\rangle$ (counts) & 0.43 & 0.43 & 0.03 & 0.03 \\
TPSD $\langle\mu(i, j)\rangle$ (counts) & 0.134 & 0.010 & 0.007 & 0.007 \\
\hline
\end{tabular}

${ }^{a}$ TPB is the typical pixel background level, calculated as the mean background of all pixels $i, j$. TPSD is the typical standard deviation of an image pixel between measurements, calculated as the mean standard deviation.

The first was extracted at the illuminating channel's modulation frequency and was used as a measure of the signal for that channel $\left(I_{\text {source }}\right)$. The second speckle pattern was extracted at the frequency corresponding to the modulation frequency used for the second illuminating channel, were it present. This contained information consisting of cross talk from the illuminating channel and contributions from the camera noise $\left(I_{\text {cross }}\right)$. The ratio of the mean intensities in $I_{\text {cross }}$ to $I_{\text {source }}$ can be used as a measure of the cross talk. Overall, cross talk was measured to be an order of magnitude higher than the camera noise floor, with the cross talk between channels, defined as the ratio of power leaked over the power remaining in the originating channel, measured to be $1.5 \%$ and $2.0 \%$ from channels one and two, respectively. This compares favorably with PDM schemes, where a cross talk of $20 \%$ has been reported [10]. Possible causes of cross talk are mixing of higher-order harmonics or overlap of peaks from different images.

\section{Application to In-Plane Shearography}

A two-component, in-plane shearography system was configured to demonstrate the use of FDM in a practical shearography system. The experimental configuration is shown in Fig. $\underline{4}$. Two illumination

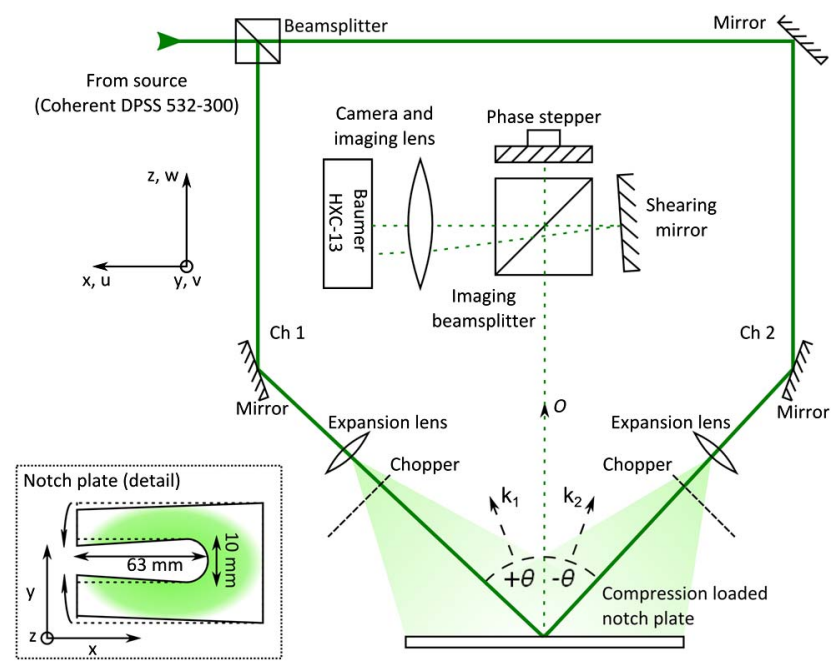

Fig. 4. (Color online) In-plane shearography configuration with beam choppers to facilitate the frequency-division multiplexing of the two channels. Angles $\pm \theta$ were $\pm 45^{\circ}$, making $k_{1}=22.5^{\circ}$ and $k_{2}=-22.5^{\circ}$ from the observation vector. Inset: notched-plate test object as viewed by the system; a $40 \mathrm{~mm}^{2}$ area was viewed around the fixed end of the notch. Loading involves compressing the two distal ends together, as shown, using a screw. channels were created by splitting equally the output from the laser (Coherent DPSS 532-300), operating with an output power of $200 \mathrm{~mW}$, into two illumination beams that had the same polarization state. The beams were expanded to illuminate the test object's surface and arranged to illuminate it from equal but opposite angles in the $x-z$ plane.

This provided sensitivity vectors, $k_{1}$ and $k_{2}$, of equal magnitude and allowed the separation of the in-plane and out-of-plane components of surface displacement gradient, as per Eqs. (2) and (יㅜ). The illumination intensity on the object surface was adjusted by varying the divergence of the transmitted beams from each channel through selection of appropriate lenses to ensure that the camera did not saturate when both channels were incident on the surface, and also to ensure that both illumination channels had similar intensity to each other. Distinct periodic intensity modulations were applied to the two channels by placement of beam choppers near the focus of the microscope objective lenses used in expanding the beams. These square-wave modulations should theoretically produce a comb of frequencies in the power spectrum, which consists of a peak at the fundamental modulation rate and then peaks of successively decreasing height at every odd harmonic. However, in practice, power was also found to have leaked into the even harmonics. It was possible to avoid the emergence of harmonic peaks by modulating at frequencies with higher harmonics that lay beyond the Nyquist frequency. Modulation frequencies were selected to avoid the fundamental component from one modulation frequency overlapping with harmonic content from the other modulation frequency, and to allow a high number of modulation cycles to be sampled while still fulfilling the Nyquist condition. Thus they were set at 140 and $175 \mathrm{~Hz}$ for channels 1 and 2, respectively, as shown in Fig. 5 .

A Michelson interferometer was used to provide a lateral image shear of $3 \mathrm{~mm}$, applied along the $y$-axis by tilting the mirror of one arm of the interferometer. The mirror of the other arm was mounted on a piezo actuator to provide longitudinal translation of the mirror, allowing use of temporal phase stepping [16]. A telephoto imaging lens arrangement was positioned between the camera (Baumer HXC-13) and the interferometer to image the surface of the object. The object was positioned $0.75 \mathrm{~m}$ from the front element of the lens. The camera was configured to use a subregion of $600 \times 600$ pixels and a framerate of $430 \mathrm{fps}$ at 8 bit depth, which allowed the capture 


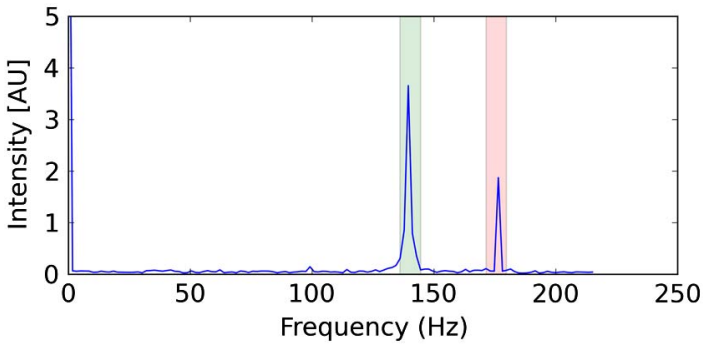

Fig. 5. (Color online) Mean power spectrum of an image bank containing two peaks corresponding to the frequencies of the modulation applied to the two channels. The truncated peak at $0 \mathrm{~Hz}$, corresponding to the background light, reached an intensity of 11,500 AU. The shaded regions show the extent of the two window functions used in discriminating channels during demultiplexing. The peaks had different heights due to a difference in intensities between the two illuminating beams.

of 256 frames in the image time series while ensuring that there were sufficient signal levels in each frame.

The test object used was a compression-loaded notch in an opaque Perspex block that produced predominantly in-plane deformations, suitable to produce responses detectable with the twocomponent in-plane shearography system. Loading was achieved using a screw on the end of the notch that was turned and pulled the ends together.

The five-frame temporal phase-stepping algorithm shown in Eq. () [21] was used to evaluate the phase:

$$
\phi=\tan ^{-1}\left[\frac{2\left(I_{2}+I_{4}\right)}{2 I_{3}-I_{5}-I_{1}}\right],
$$

This was selected due to its tolerance toward many systematic detector errors [16]. The experimental procedure was to capture five time series, one at each of the five phase steps, apply a load to the object, and then capture another five phase-stepped time series. An additional image was captured before each phasestep time series to obtain time-division multiplexed (TDM) data. Doing so allowed for the direct comparison of the performances of TDM and FDM approaches under the same loading conditions.

To process the modulated data, the time series were processed as described above. The peaks in the power spectrum at the fundamental frequencies were selected using bandpass filters centred on the fundamental frequencies with a width of $10 \mathrm{~Hz}$ to ensure capture of the entire peak. The reconstructed speckle patterns were then converted to a phase map for the loaded state via the phase-stepping algorithm, filtered with five passes of a sine-cosine filter [22], and unwrapped using the "iso-phase unwrapping" algorithm [23]. Maps of the change of phase between the reference and loaded states were then calculated, which can be described in the form of Eqs. (8) and (9) for the two channels of an in-plane shearography system:

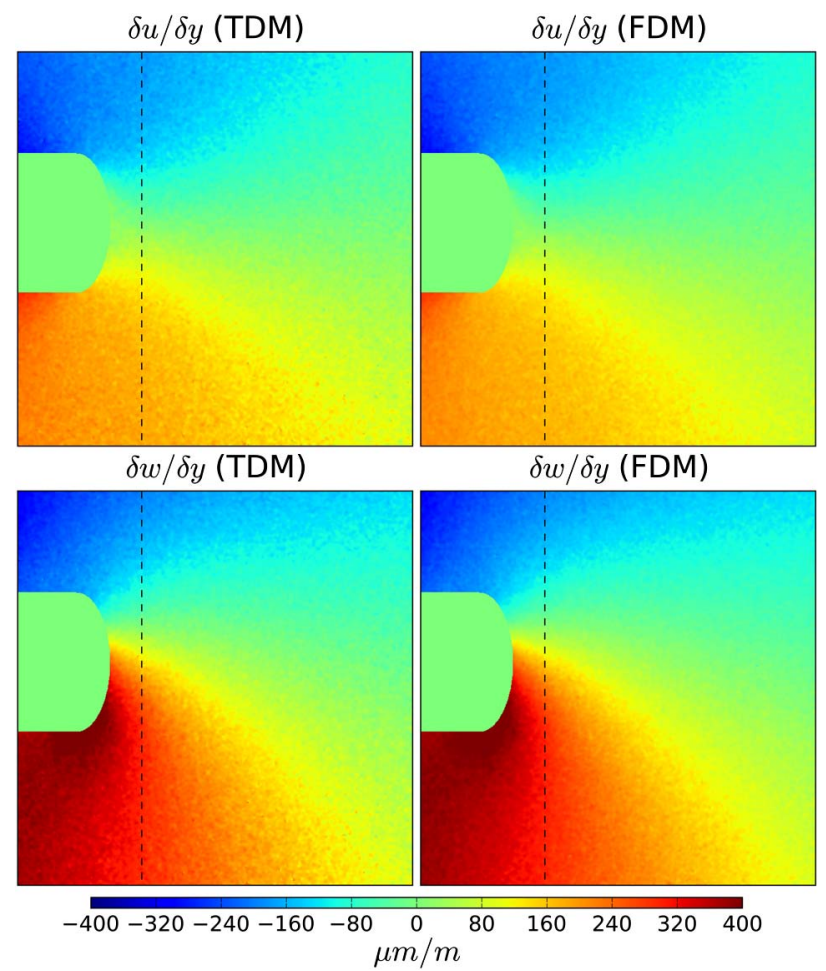

Fig. 6. (Color online) In-plane (top) and out-of-plane (bottom) displacement gradient components of a $41 \mathrm{~mm}$ by $41 \mathrm{~mm}$ area around a compression-loaded notch in a Perspex plate. The left column shows results obtained using TDM, and the right column shows those obtained using FDM. The dashed lines indicate the location of the line plots in Fig. $\underline{7}$.

$$
\begin{aligned}
& \Delta \phi_{1}=\frac{2 \pi \delta y}{\lambda}\left[k_{x} \frac{\partial u}{\partial y}+k_{z} \frac{\partial w}{\partial y}\right], \\
& \Delta \phi_{2}=\frac{2 \pi \delta y}{\lambda}\left[-k_{x} \frac{\partial u}{\partial y}+k_{z} \frac{\partial w}{\partial y}\right],
\end{aligned}
$$

The values in these two-phase change maps were then processed by subtraction to reveal the in-plane

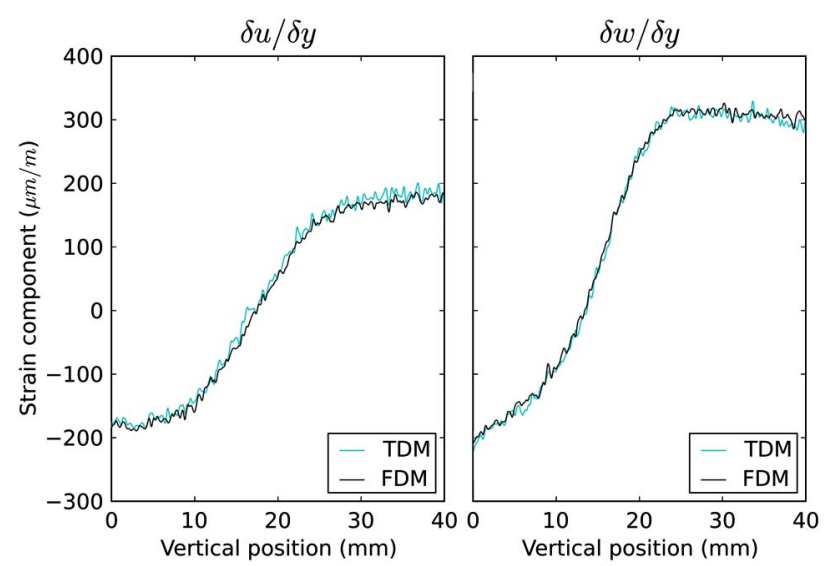

Fig. 7. (Color online) Vertical profile taken from the displacement gradient maps shown in Fig. $\underline{6}$, near the apex of the notch. The left plot shows the profiles for the in-plane component, and the right plot is the same for the out-of-plane component. 


\begin{tabular}{|c|c|c|}
\hline Method & Pros & Cons \\
\hline WDM & $\begin{array}{l}\text {-Use of full image sensor } \\
\text { - Simultaneous image capture }\end{array}$ & $\begin{array}{l}\text {-Different wavelength sources required } \\
\text { - Cross talk from wavelength selection }\end{array}$ \\
\hline PDM & $\begin{array}{l}\text {-Use of full image sensor } \\
\text { - Simultaneous image capture }\end{array}$ & $\begin{array}{l}- \text { Cross talk from polarization optics } \\
\text { - Not suitable on depolarizing surfaces }\end{array}$ \\
\hline SM & $\begin{array}{l}\text { - Simultaneous image capture } \\
\text { - No cross talk }\end{array}$ & $\begin{array}{l}\text { - Uses subregions of image sensor or multiple sensors. } \\
\text { - Requires precise interimage alignment }\end{array}$ \\
\hline FDM & $\begin{array}{l}\text { - Use of full image sensor } \\
\text { - Simultaneous image capture } \\
\text { - Inherent noise suppression } \\
\text { - Simple implementation by } \\
\text { beam chopping } \\
\text { - Low cross talk }\end{array}$ & $\begin{array}{l}\text { - Image time series required. } \\
\text {-Dynamic range reduced by increasing number of channels }\end{array}$ \\
\hline
\end{tabular}

${ }^{a}$ TDM, time-division multiplexed; WDM, wavelength-division multiplexed; PDM, polarization-division multiplexed; SM, spatially multiplexed; FDM, frequency-division multiplexed. Italic entries are considered unique to the FDM technique.

strain distribution [Eq. (2)], and by summing to reveal the out-of-plane strain distribution [Eq. (3)].

The time-division multiplexed images were processed by passing them directly to the phasestepping algorithm, and from there following the same processing steps as used for obtaining the two displacement gradient components with the FDM data.

The in-plane and out-of-plane displacement gradient components on the notch plate are shown in Fig. 6 for both TDM and FDM. The strain maps for both in-plane and out-of-plane strains are qualitatively similar for the TDM and FDM cases, and a quantitative comparison showed that the FDM results agreed to within $1.5 \%$ of the TDM method across the field of view. An example vertical cross section of the data shown in Fig. 6 has been plotted in Fig. 7, showing the components of strain from beside the notch region. Both components of strain and both multiplexing techniques represented in Fig. 6 are plotted, and show the agreement between the TDM and FDM results.

The results demonstrated that the FDM technique can be applied successfully to a multicomponent shearography system and that the results are comparable to the established TDM technique.

\section{Conclusions}

The development of a new method to multiplex the channels in a shearography system onto a single camera for simultaneous capture has been presented. The FDM method allows multiplexing of the channels using the full image sensor resolution simultaneously for each channel, with low cross talk between channels and inherent noise suppression. The relative advantages of the FDM technique over other multiplexing techniques are summarized in Table 2. Measurements undertaken using the
FDM technique have been shown to be comparable to the established TDM technique, agreeing to within 1.5\%. Cross talk between the multiplexed speckle images was shown to be less than $2 \%$ of the power in the originating channel, and camera noise at the demultiplexing frequencies was reduced by a factor in excess of an order of magnitude compared to that of a single frame.

It is expected that the multiplexing capacity can be increased to allow for the multiplexing of additional channels, and for multiplexing orthogonal shear directions. This would allow for the simultaneous capture of all of the required information necessary to calculate complete surface strain.

The authors acknowledge the support of the Engineering and Physical Sciences Research Council (EPSRC) UK, via grant EP/G033900/1 and a studentship for IAB.

\section{References}

1. D. Francis, R. P. Tatam, and R. M. Groves, "Shearography technology and applications: a review," Meas. Sci. Technol. 21, 102001 (2010).

2. M. Kalms and W. Osten, "Mobile shearography system for the inspection of aircraft and automotive components," Opt. Eng. 42, 1188-1196 (2003).

3. U. Paul Kumar, M. P. Kothiyal, and N. Krishna Mohan, "Microscopic TV shearography for characterization of microsystems," Opt. Lett. 34, 1612-1614 (2009).

4. W. Steinchen and L. Yang, Digital Shearography (SPIE, 2003).

5. S. W. James and R. P. Tatam, "3D shearography for surface strain analysis," Proc. SPIE 3783, 247-256 (1999).

6. T. O. H. Charrett, D. Francis, and R. P. Tatam, "Quantitative shearography: error reduction by using more than three measurement channels," Appl. Opt. 50, 134-146 (2011).

7. D. T. Goto and R. M. Groves, "Error analysis of 3D shearography using finite-element modelling," Proc. SPIE 7718, 771816 (2010).

8. D. Francis, S. W. James, and R. P. Tatam, "Surface strain measurement of rotating objects using pulsed laser shearography with coherent fibre-optic imaging bundles," Meas. Sci. Technol. 19, 105301 (2008). 
9. R. M. Groves, S. W. James, and R. P. Tatam, "Full surface strain measurement using shearography," Proc. SPIE 4448, 142-152 (2001).

10. R. M. Groves, S. W. James, and R. P. Tatam, "Polarizationmultiplexed and phase-stepped fibre optic shearography using laser wavelength modulation,” Meas. Sci. Technol. 11, 1389-1395 (2000).

11. R. Kästle, E. Hack, and U. Sennhauser, "Multiwavelength shearography for quantitative measurements of two-dimensional strain distributions," Appl. Opt. 38, 96-100 (1999).

12. Y. Y. Hung, "Shearography: a new optical method for strain measurement and nondestructive testing," Opt. Eng. 21, 213391 (1982).

13. S. W. James, R. M. Groves, and R. P. Tatam, "Surface strain characterisation using time-division-multiplexed 3D shearography," Proc. SPIE 4101, 121-129 (2000).

14. H. A. Aebischer and S. Waldner, "Strain distributions made visible with image-shearing speckle pattern interferometry," Opt. Lasers Eng. 26, 407-420 (1997).

15. S. W. James, R. P. Tatam, and R. L. Elder, "Design considerations for a three dimensional fiber optic laser Doppler velocimeter for turbomachinery applications," Rev. Sci. Instrum. 68, 3241-3246 (1997).
16. K. Creath, "Temporal phase measurement methods," in Interferogram Analysis: Digital Fringe Measurement Methods, D. W. Robinson and G. T. Reid, eds. (IOP, 1993), pp. 94-140.

17. Python Software Foundation, "Python," http://www.python .org.

18. T. O. H. Charrett, "Python Toolkit," http://pythontoolkit .sourceforge.net/.

19. "SciPy: open source scientific tools for Python" (2001), http://www.scipy.org.

20. M. Frigo and S. G. Johnson, "Fastest Fourier transform in the west," http://www.fftw.org.

21. J. Schwider, R. Burow, K.-E. Elssner, J. Grzanna, R. Spolaczyk, and K. Merkel, "Digital wave-front measuring interferometry: some systematic error sources," Appl. Opt. 22, 3421-3432 (1983).

22. A. Aebisher and S. Waldner, "A simple and effective method for filtering speckle-interferometric phase patterns," Opt. Commun. 162, 205-210 (1999).

23. M. A. Herráez, M. A. Gdeisat, D. R. Burton, and M. J. Lalor "Robust, fast, and effective two-dimensional automatic phase unwrapping algorithm based on image decomposition," Appl. Opt. 41, 7445-7455 (2002) 